\title{
Spontaneous Perforation of the Colon - A possible Third Classification
}

\author{
Amanda Prado ${ }^{1}$ (1), Ricardo Pedrini Cruz * * (])
}

\section{Dear Editor,}

The article by Moon et al. ${ }^{1}$ brings a very important differential diagnosis of spontaneous perforation of the colon (SPC), pathology seldom recognized by surgeons. The frequency of this emergency condition may be underestimated and consequently rare reported. SPC was first described in $1894^{2}$, as being classified as stercoral or idiopathic perforation ${ }^{2-4}$. Stercoral perforation of the large bowel is a rare event (with less than 100 cases reported in the literature), and idiopathic perforation is even more rarely reported ${ }^{4}$.

A 46 years old white man was admitted complaining of abdominal pain and distension in the past 2 days, partially relieved with powdered magnesium sulfate. The pain worsened in the last 24 hours, without elimination of flatus or feces. He had a history of arterial hypertension (treated with captopril and hydrochlorothiazide), alcohol and tobacco consumption, and chronic constipation. Stercoral perforation usually occurs in patients with chronic constipation ${ }^{1,5}$. The patient reported constipation in the last 30 years, worsening in the last 9 years. During this period, daily laxative use (powdered magnesium sulfate and senna leaves compound) was necessary for symptom alleviation. He denied previous hospitalization or surgery. In the admission, the patient had no fever, presenting distended abdomen, diffuse abdominal pain and rebound tenderness on physical exam. Laboratory tests showed 9600 leukocytes with $41 \%$ of younger white cells (bands and metamyelocytes). An abdominal CT scan was performed, showing a sigmoid wall perforation, no fecaloma, and pneumoperitoneum (Figure 1). A laparotomy was indicated, showing fecal peritonitis and sigmoid colon perforation, treated with Hartmann's procedure. No tumor, fecaloma, or diverticulosis were found. The sigmoid colon had a hypertrophic muscular wall and a narrowed lumen with a $0.5 \mathrm{~cm}$ circular perforation on the antimesenteric tenia, with necrotic borders. Pathology analysis evidenced an area of necrosis on the antimesenteric sigmoid border, precisely in the colic tenia. There were no signs of previous pathologies, such as ulcers, or diverticula. Ciprofloxacin, metronidazole and fluconazole were used for 15 days. He was discharged without any complication. Colonoscopy was performed without

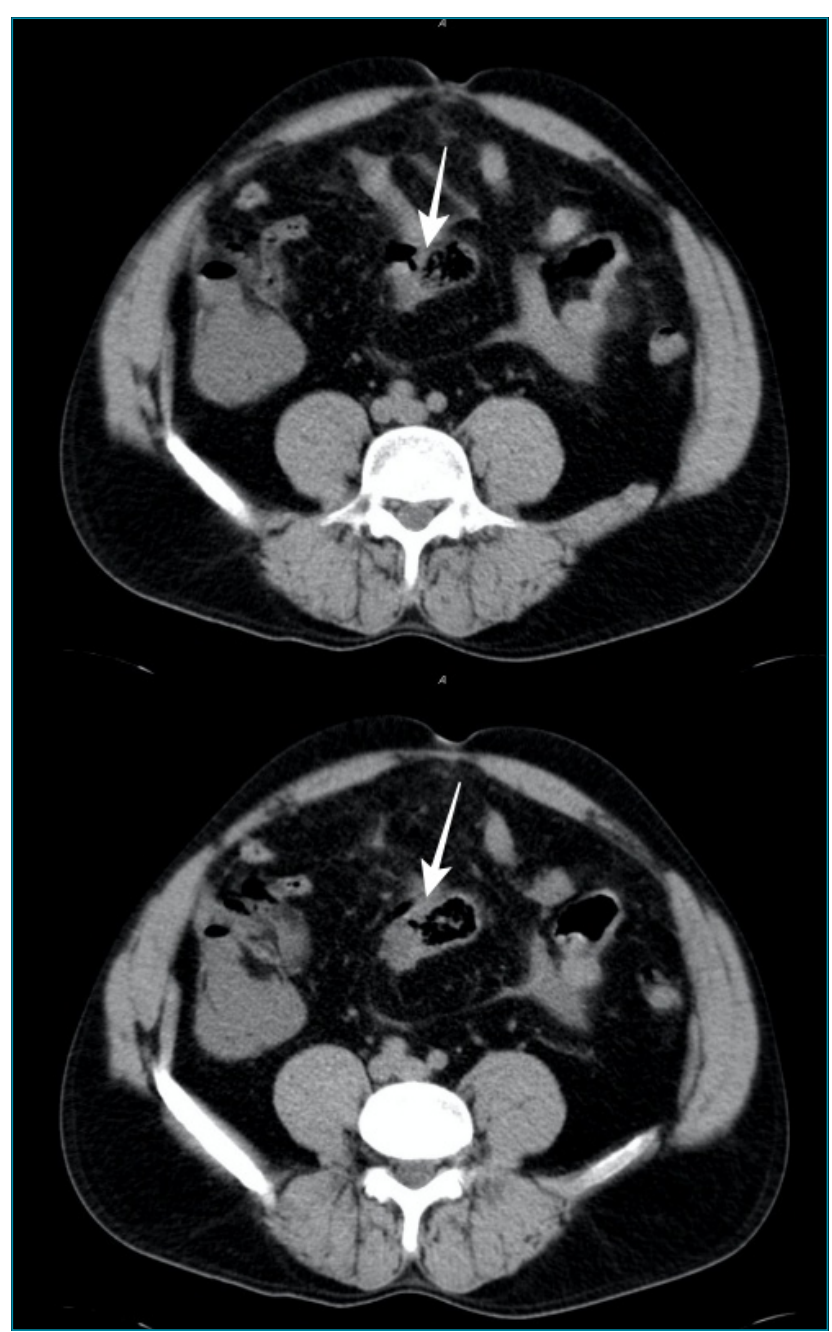

Figure 1. Abdominal CT showing a sigmoid wall perforation, no fecaloma, and pneumoperitoneum (white arrows).

${ }^{1}$ Hospital Nossa Senhora da Conceição, Department of Surgical Oncology - Porto Alegre (RS), Brazil.

*Corresponding author: ricardoc@ghc.com.br

Conflicts of interest: the authors declare there are no conflicts of interest. Funding: none.

Received on October 15, 2020. Accepted on October 20, 2020. 
any abnormality. The bowel transit was reconstructed 2 months after the first surgery. He reported improvement of constipation after sigmoid resection, without any constipation thereafter.

SPC is defined as a sudden perforation of the normal colon in the absence of tumors, diverticulosis, or external injury ${ }^{5}$. Stercoral perforation occurs more frequently on the antimesenteric border of the rectosigmoid, an area more prone to ischemia because of anatomical vascularization ${ }^{2}$. The SPC has been classified as stercoral and idiopathic ${ }^{1,2}$. Stercoral perforations are those with rounded shape perforation with more than $1 \mathrm{~cm}$ in diameter; the colon should be full of stool, which diffuses to the abdominal cavity through the perforation; microscopical examination should evidence ischemia and necrosis of colonic mucosa leading to feculent ulcer and acute inflammatory reaction surrounding the perforation site; and external injury or other diseases such as obstruction, tumors, and diverticulosis must be excluded. The idiopathic SPC occurs due to asymmetrical distribution of intraluminal pressure at the pelvirectal angle in the absence of obvious impacted fecal matter ${ }^{4,5}$. According to Kasahara et al. ${ }^{6}$ idiopathic SPC has a linear perforation and the feculent ulcer cannot be seen at microscopic examination. Other characteristics are a clear mucosal edge that does not extend to the serosa, and a regular broken end of the muscular layer5. Our patient had chronic constipation with laxative abuse, a narrowed lumen with a hypertrophic muscular wall in the sigmoid colon, and necrotic $0.5 \mathrm{~cm}$ diameter perforation on the antimesenteric tenia without fecaloma. Although a higher association with a layered-enhancing wall thickening was described in the stercoral SPC ${ }^{1}$, the absence of a fecaloma and a colon perforation of only $0.5 \mathrm{~cm}$ without surrounding acute inflammatory reaction in the perforation site, prevent its classification as stercoral SPC. The idiopathic SPC classification is neither possible: no linear perforation was evidenced and there is no evidence that an asymmetrical distribution of intraluminal pressure at the pelvirectal angle could be a reasonable explanation of the perforation. Moreover, the patient had bowel wall hypertrophy with a narrowed lumen, aspects not present in the previous classifications.

Chronic constipation has an important association with indiscriminate laxative use. There is some evidence about colon ischemia by hyperosmotic laxatives ${ }^{7}$, probably by the rapid shift of fluids from the vascular space of the colonic wall circulation into the luminal space resulting in local hypoperfusion. The mechanism of bowel ischemia by stimulant laxatives is a combination of colon motility stimulation with the increase of bowel luminal pressure, decreasing consequently the colonic perfusion by splanchnic circulation compression ${ }^{7}$. Our patient used senna leaves compound (a nonspecific bowel laxative stimulant/irritant) and powdered magnesium sulfate (a luminally active laxative agent). These laxatives may have contributed to impaired perfusion of the colon wall, leading to its ischemia and spontaneous perforation.

In conclusion, there is probably another pathophysiological mechanism that differs from those two described in the literature. The SPC associated with chronic constipation and laxative abuse is characterized by a narrowed sigmoid lumen, hypertrophic bowel wall musculature, no fecaloma, and perforation on the antimesenteric taenia of sigmoid, in the absence of other diseases (as tumors, diverticulosis, ulcers, or infection signs). Many stercoral SPC reported in the literature may be in fact, an SPC associated with laxative abuse.

\section{AUTHORS" CONTRIBUTIONS}

RPC: Conceptualization, Formal Analysis, Investigation, Methodology, Project Administration, Supervision, Validation, Visualization, Writing - Original Draft, Writing - Review \& Editing. AP: Data Curation, Methodology, Project Administration, Validation, Visualization, Writing - Original Draft.

\section{REFERENCES}

1. Moon JY, Hong SS, Hwang J, Lee HK, Choi KC, Cha H, et al. Differentiation between stercoral perforation and colorectal cancer perforation. Rev Assoc Med Bras (1992). 2019;65(2):191-7. https://doi.org/10.1590/1806-9282.65.2.191

2. Maurer CA, Renzulli P, Mazzucchelli L, Egger B, Seiler CA, Büchler $\mathrm{MW}$. Use of accurate diagnostic criteria may increase incidence of stercoral perforation of the colon. Dis Colon Rectum. 2000;43(7):991-8. https://doi.org/10.1007/BF02237366

3. Zachariah SK, Raja N. Spontaneous perforation of the colon and hypothyroidism: report of a case and review of literature. Gastroenterology Res. 2010;3(3):147-9. https://doi.org/10.4021/ gr2010.06.212w
4. Al Shukry S. Spontaneous perforation of the colon clinical review of five episodes in four patients. Oman Med J. 2009;24(2):13741. https://doi.org/10.5001/omj.2009.30

5. Yang B, Ni HK. Diagnosis and treatment of spontaneous colonic perforation: analysis of 10 cases. World J Gastroenterol. 2008;14(28):4569-72. https://doi.org/10.3748/wjg.14.4569

6. Kasahara $Y$, Matsumoto $H$, Umemura $H$, Shirafa $S$, Kuyama T. Idiopathic perforation of the sigmoid colon in Japan. World J Surg. 1981;5(1):125-30. https://doi.org/10.1007/BF01657857

7. Vodusek Z, Feuerstadt P, Brandt LJ. Review article: the pharmacological causes of colon ischaemia. Aliment Pharmaco Ther. 2019;49(1):51-63. https://doi.org/10.1111/apt.15052 